\title{
Design, fabrication and electromagnetic testing of multi-layered multi- permittivity radar absorbing structural composite for stealth applications
}

\author{
H.B. Baskey*, T.C. Shami, A.K. Dixit, Rudresh Kumar, Sumit Kumar and N.Eswara Prasad \\ Defence Materials and Stores Research and Development Establishment (DMSRDE) \\ G. T. Road, Kanpur-208013 (INDIA) \\ *Email: himanshudmsrde@gmail.com
}

The Radar Absorbing Structures (RAS) plays an important role in the stealth technology [13] for various weapon systems for mitigating the incident electromagnetic waves. The application of RAS reduces the radar cross section of the targets and thus reduces its detectability against enemy radar system. The high strength to weight ratio of a composite as a structural component results in improved capabilities, such as increased payload, higher top speed, greater range, and reduced fuel usage in aerospace applications. Furthermore RAS possess both structural as well as electromagnetic absorbing properties. In this proposed paper a multilayer multi-permittivity RAS have been designed for $\mathrm{X}$ band (8.2$12.4 \mathrm{GHz}$ ) frequency region. The electromagnetic design and the optimization RAS composite was carried out using three dimensional electromagnetic simulator.

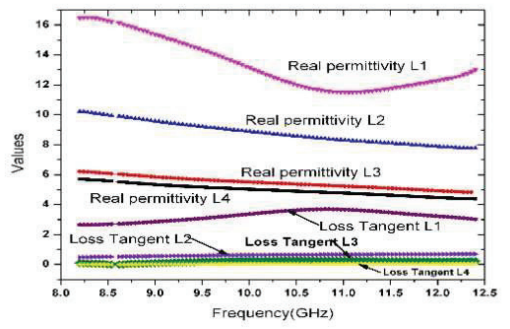

Figure 1: Real permittivity and loss tangent of various layer of RAS

The various electromagnetic properties of the RAS laminates have been achieved by optimizing various weight percentages of lossy fillers e.g. carbon black, carbon fibers in the epoxy matrix and glass fabrics. The real permittivity and the loss tangent of the various layers of the composite are given in the Figure 1. It is to be noted that the highest permittivity is achieved for the layer L1 (17.2-14),similarly highest loss tangent is also achieved for the same layer. The gradation in the permittivity and the loss tangent is achieved for the various layer of the stacks in order to have the effective attenuation of the electromagnetic energy. The fabrication of the prototype monolithic composite was carried out using optimized four layer gradient RAS laminates using vacuum bagging technique. The fabricated structural composite was characterized for radar absorbing properties using Free Space Measurement Technique as shown in the Figure 2a. The electromagnetic performance of the fabricated composite shows greater than $10 \mathrm{~dB}$ return loss (90\% absorption) of the fabricated RAS in the entire $X$ band frequency region as shown in the Figure $2 b$. The developed RAS exhibits excellent radar absorbing and mechanical properties.
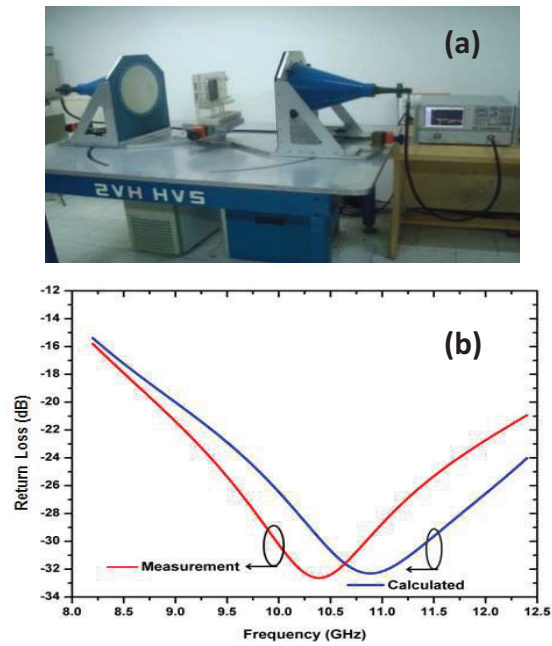

Figure 2: (a) Experimental set up for the measurement of return loss (b) Comparison of the simulated and the measured return loss

\section{References}

1. W. F. Bahret, "The beginnings of stealth technology," IEEE Trans. Aerosp. Electron. Syst., vol. 29, no. 4, pp. 1377-1385, Oct. 1993

2. Knott EF, Schaeffer JF, Tuley M. Radar Cross Section. 2nd ed. Boston, London:Artech House; 1993.

3. Vinoy K J,Jha R M. Radar Absorbing Materials, Boston, MA:Kluwer-Academic 